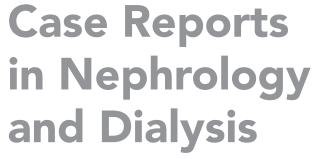

Case Reports and Dialysis

\title{
Idiopathic Spontaneous Rupture of Renal Pelvis in a Single Functioning Kidney
}

\author{
Maciej Tylski Katarzyna Muras-Szwedziak Michał Nowicki \\ Department of Nephrology, Hypertension and Kidney Transplantation, Medical University \\ of Lodz, Lodz, Poland
}

\section{Keywords}

Spontaneous rupture of renal pelvis - Single functioning kidney · Urine extravasation ·

\section{Urinoma}

\begin{abstract}
Spontaneous rupture of renal pelvis (SRRP) is a rare condition resulting in an extravasation of urine into retroperitoneal space. Due to the uncharacteristic symptoms, often mimicking renal colic, its diagnosis may be complicated. Herein, we report a case of a 73-year-old male with a solitary functioning kidney who presented with malaise and right-sided abdominal pain, rapidly followed by anuria. Laboratory tests showed the signs of AKI. Contrast-enhanced CT performed soon after the admission showed nonspecific abnormalities in the right middle abdomen suspected to be either inflammatory infiltration or surgical scarring. Symptomatic treatment was started, and an acute hemodialysis treatment was commenced. After a temporal improvement, the patient's general condition worsened significantly, with exacerbated pain and massive increase in plasma creatinine. A second contrast-enhanced CT was performed with an addition of urography phase, revealing the extravasation of the contrast media in the location suggesting the rupture of the renal pelvis. The patient was treated successfully by the placement of a double-J ureteral stent into the ureter. Usually, a clear etiology of SRRP can be determined, that is, urinary tract obstruction, but in this case, we could not find a definite cause. It is important to remember that in the presence of a nonspecific abdominal pain and laboratory signs of AKI, a rare cause like SRRP should be taken into consideration. Performing a contrast CT scan with urography phase can save time in establishing a diagnosis and enable immediate urological intervention.
\end{abstract}

\section{Karger $\stackrel{2}{*}$}




\section{Introduction}

A spontaneous renal collecting system rupture is defined as the nontraumatic extravasation of urine. A review of the documented cases shows the renal fornix to be the most common rupture site; ruptures of renal pelvis or ureteropelvic junction are very rare occurrences [1]. The disruption of the pelvicalyceal system may result from an elevated intrapelvic pressure, typically secondary to urinary tract obstruction [2,3]. Ureteric calculi are reported to be the leading cause of this complication; less common causes include malignancies, pregnancy, posterior urethral valves, ureteropelvic junction obstruction, and medications [4]. Very few reports describe the cases of spontaneous renal collecting system rupture without any identifiable underlying pathology [5].

Below, we present the case of an adult patient with spontaneous rupture of renal pelvis (SRRP) of a single functioning kidney [6]. In this case, in spite of numerous conditions predisposing to urinary tract obstruction, no definitive cause of rupture has been established.

\section{Case Presentation}

A 73-year-old male patient presented to the surgical ward with a general malaise and right-sided abdominal pain that had presented for 2 days. His past medical history included left nephrectomy due to nephrolithiasis at the age of 48, CKD of unknown etiology, and right hemicolectomy with adjuvant chemotherapy due to colonic adenocarcinoma 5 years prior to the current hospitalization. The physical examination at the admission to our hospital revealed no signs of peritonitis. Goldflam sign was absent. The abdominal ultrasound revealed no pathology. A noncontrast abdominal CT scan was performed, and a suspicion of either inflammatory infiltration or surgical scarring in the right middle abdomen was indicated by the radiologists. Laboratory results showed signs of AKI, with plasma creatinine $390.6 \mu \mathrm{mol} / \mathrm{L}$ on day 1 rising quickly to $647.6 \mu \mathrm{mol} / \mathrm{L}$ on the second day of hospitalization, metabolic acidosis, hyponatremia (sodium $133.7 \mathrm{mmol} / \mathrm{L}$ on day 2), and hyperkalemia (potassium 5.49 $\mathrm{mmol} / \mathrm{L}$ on day 1 and $5.77 \mathrm{mmol} / \mathrm{L}$ on day 2). CRP started to increase on day 2, reaching 52.4 $\mathrm{mg} / \mathrm{L}$ on the following day. The empirical antibiotic therapy consisting of ciprofloxacin and metronidazole was administered after both blood and urine samples were taken for bacteria culture test.

Shortly after being admitted to our ward, the patient developed anuria, despite intravenous infusions of fluids and loop diuretics initiated at the hospital emergency department and continued at our ward. The kidney function impairment reflected by the raising plasma creatinine levels in subsequent days was worsening rapidly. Considering deteriorating patient's general condition and the laboratory findings, an acute daily hemodialysis treatment was started, and a decision to transfer the patient to the nephrology ward in our hospital was made.

In the nephrology ward, the pharmacotherapy was maintained with no significant modifications, and the patient's condition temporarily improved. Over the course of 3 days, serum creatinine decreased from $827.5 \mu \mathrm{mol} / \mathrm{L}$ to $403.5 \mu \mathrm{mol}$, and diuresis increased to up to $4 \mathrm{~L}$ per day. Serum electrolytes were within the normal range. Hemodialysis was deemed no longer necessary.

After 7 days of hospitalization, the patient complained of a sudden exacerbation of the abdominal pain. The pain was located in the right middle abdomen and radiated to the right iliac fossa and was accompanied by a positive Goldflam sign. At the same time, urine output started to decrease rapidly, and macroscopic hematuria had been observed before the patient

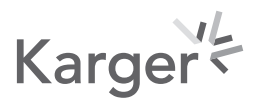




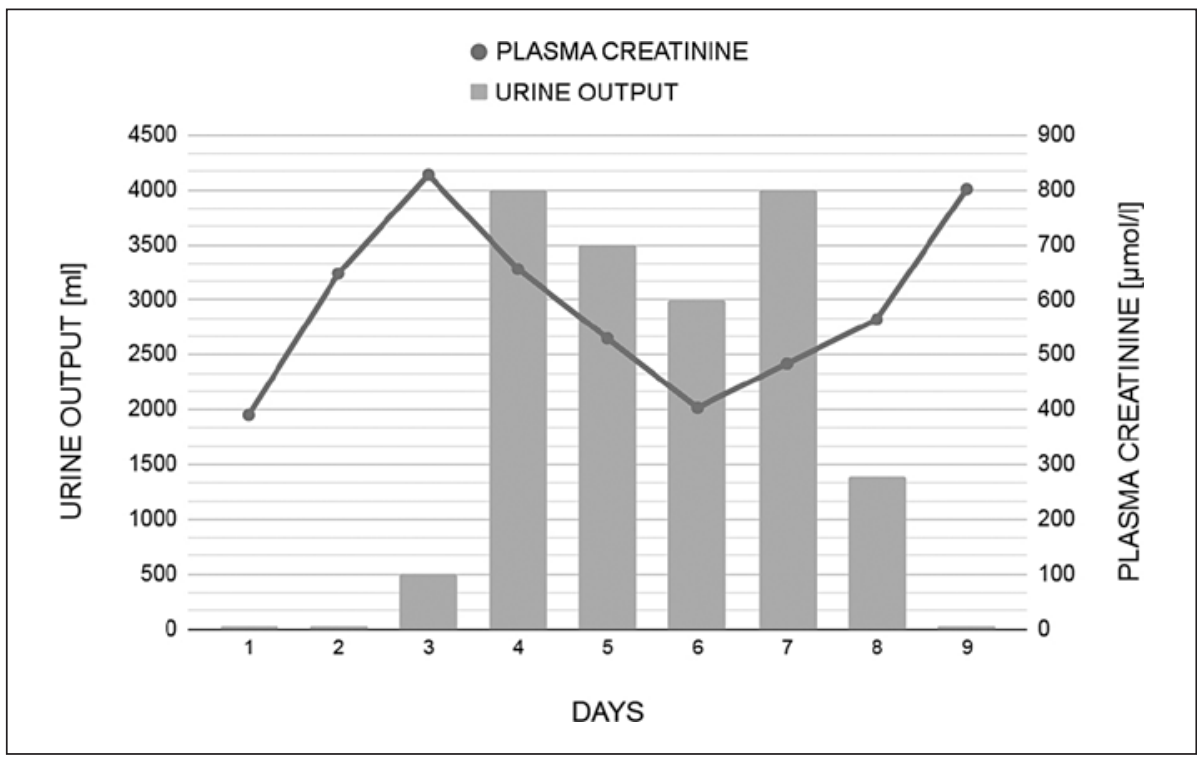

Fig. 1. The changes of serum creatinine and urine output in the first 9 days of hospitalization, before surgery.
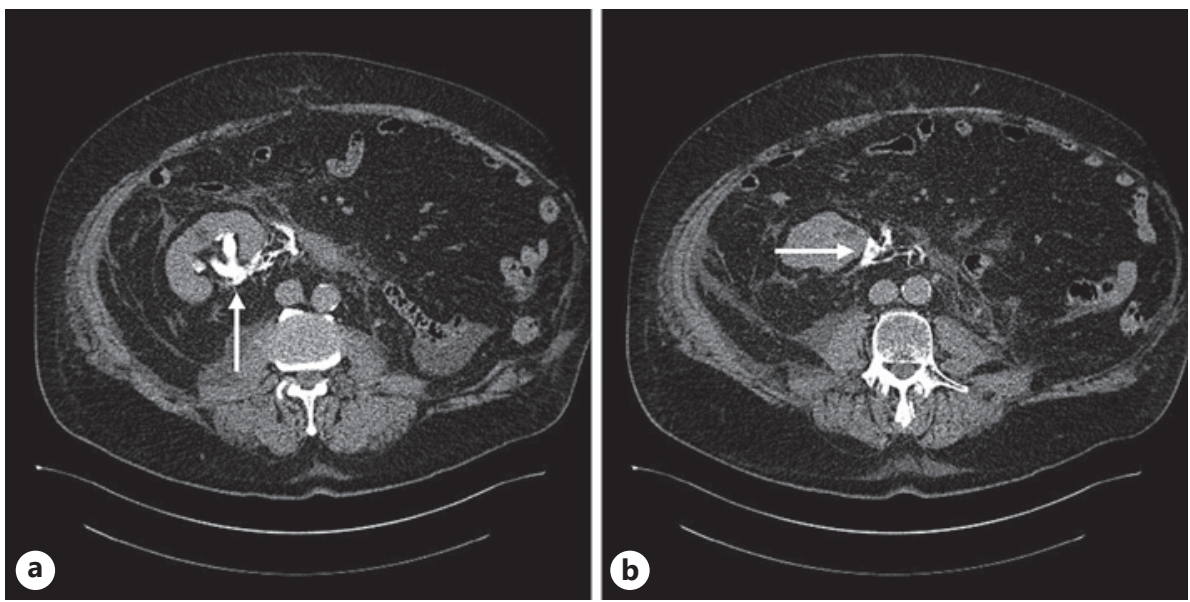

Fig. 2. Contrast-enhanced CT, urography phase. a Extravasation of the contrast medium in the proximity of the right renal pelvis. $\mathbf{b}$ Extravasation of the contrast medium alongside the right ureter.

became anuric for the second time. Serum creatinine increased quickly to $801.6 \mu \mathrm{mol} / \mathrm{L}$. The changes of serum creatinine and urine output are presented in Figure 1. Both abdominal ultrasonography and plain abdominal X-ray showed no abnormalities aside from a slight pelvicalyceal system dilation. A second contrast-enhanced CT scan was performed, this time with urography phase, revealing the extravasation of the contrast medium in the proximity of the right renal pelvis (Fig. 2a) and alongside the right ureter (Fig. 2b), suggesting the rupture of the renal pelvis.

The patient had 1 acute hemodialysis session performed and then was transported to the urology ward. A cystoscopy was performed, during which a double-J ureteral stent was placed into the right ureter. After the procedure symptoms subsided, diuresis reached $5 \mathrm{~L}$ per day and plasma creatinine decreased to $296.2 \mu \mathrm{mol} / \mathrm{L}$. The patient was discharged in a good general condition, with frequent checkups at a local nephrology clinic recommended. 


\section{Discussion}

A spontaneous rupture of the renal collecting system is a rare complication that may occur in the presence of an elevated intrapelvic pressure. It can develop as the result of the urinary tract obstruction or very infrequently during a course of infection. Statistics showed that a vast majority of ruptures were caused by ureteric calculi [4]. Other less common causes may include malignant or benign tumors, pregnancy, posterior urethral valves, ureteropelvic junction obstruction, vascular extrinsic compression [4], and retroperitoneal fibrosis [7]. The retrograde manipulation of the ureter was also described as a cause of the urine collecting system rupture [4]. In case of our patient, several potential etiologies of rupture could be suspected, with urinary lithiasis being the most probable one. The patient had already been prone to the formation of renal calculi, as shown by the history of nephrolithiasis. He suffered from abdominal pain 2 days prior to the admission, which could have been a result of intermittent urinary tract obstruction by a renal calculus. The urinalysis performed right at the admission to the hospital would have provided clues whether that was the case. However, the urine sample was collected only for urine culture before the patient became anuric. The importance of urinalysis with urine microscopy cannot be overestimated in renal patients, especially when dealing with AKI of unknown etiology. It should always be performed in the first place - as our case illustrates, it may be a matter of time before a clinician is no longer able to obtain urine for any of the laboratory tests. As a result, even though there was a moderate possibility of urinary lithiasis, upon close retrospective examination by our team, no definitive cause could be eventually identified.

The urine leak localizes itself in the place of the lowest tensile strength, that is, the fornix of renal calyx in most cases [8]. The extent of the rupture is considered to be selflimiting $[3,9]$, as the extravasation of urine decreases intrapelvic pressure, preventing other structures of the collecting system from being damaged. Renal forniceal rupture can be treated conservatively and poses little risk of sequelae [9]. In comparison, the SRRP occurs very rarely and presents a greater threat and therefore always requires medical intervention. It happens typically in a kidney affected by a preexisting disease $[5,8,9]$ that has weakened the structure of the renal pelvis wall. We can confirm that our patient suffered from CKD of his solitary functioning kidney prior to the rupture, although the CKD etiology is unclear.

The clinical manifestations of SRRP mimic the renal colic in most cases. The pain tends to be of sudden onset and often radiates to the inguinal region [5], as was the case in our patient. A pain intensity may differ in individual cases, ranging from mild discomfort in the abdominal area to persistent pain accompanied by the signs of an acute abdomen $[9,10]$. Those symptoms are primarily associated with the extravasation of urine causing thereby a chemical peritonitis [9]. Additionally, the patients with SRRP often complain of acute gastrointestinal symptoms, like nausea and vomiting. This results not only from the aforementioned irritation of the peritoneum, but also from the physical pressure of the mass of forming urinoma on the wall of the intestines [9]. Uremia can also be a contributing factor to developing gastrointestinal symptoms. In some cases, including ours, macroscopic hematuria can be seen [5].

SRRP manifests mainly as an acute rise in plasma creatinine. However, this does not reflect a real AKI - but a "pseudo renal failure" caused by the reabsorption of creatinine [2, $10,11]$. Resulting creatinine overload overcomes renal filtration power, even in a scenario of SRRP where the contralateral kidney has fully preserved filtration function and there is no obstruction in its collecting system [11]. In patients with unilateral renal failure or, as it was the case with our patient, with a single functioning kidney, the plasma creatinine can quickly rise to very high levels. Increased plasma urea level, hyperkalemia, hyponatremia, and meta-

\section{Karger's}


Case Reports in Nephrology and Dialysis

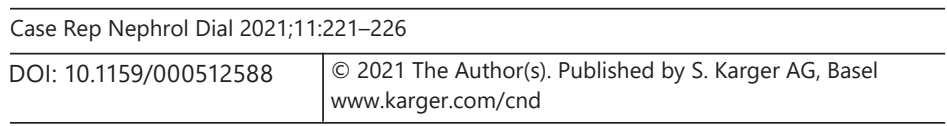

Tylski et al.: Idiopathic Spontaneous Rupture of Renal Pelvis

bolic acidosis may be present in patients with SRRP, since urea, electrolytes, and other ions diffuse according to the concentration gradient between plasma and extravasated urine in the same way creatinine does [10]. All those laboratory findings were present in our patient at admission, although electrolyte disturbances were moderate compared to the increase in plasma creatinine and urea. It should be noted that the increase in plasma creatinine was exceptionally high, even for a patient with failure of a single functioning kidney. Considering that, an element of parenchymal AKI might have played an additional role in the clinical course.

Due to the symptoms mimicking renal colic and other acute abdominal disorders, the patients with SRRP are most likely to be examined in the first place with a plain abdominal radiograph and an abdominal ultrasound. Those imaging techniques are usually not helpful in case of SRRP [12], although abdominal ultrasonography can show hydronephrosis and/or perinephric fluid collection, which may provide directions to extended diagnostics $[3,9]$. The diagnosis can be established using the intravenous pyelography and/or contrast-enhanced abdominal CT scan, with the latter becoming now a preferred method. The CT scan is best performed with a urography phase. Aside from revealing the extravasation of contrast media in the retroperitoneal, perinephric, or peripelvic space [3], it may also show the exact location of rupture [9].

The primary treatment of SRRP consists of the immediate urinary diversion [12] and broad-spectrum antibiotic [8]. The placement of a double-J ureteral stent is currently the method of choice; percutaneous nephrostomy is seldom performed; however, it is a viable urinary diversion technique in case of complicated ruptures [3]. The cause of elevated intrapelvic pressure should be identified and treated adequately if present. Open surgery is indicated only in the most difficult cases with the extensive rupture of the renal pelvis or if necessary due to the pathology that resulted in the urinary tract obstruction $[3,12]$.

\section{Statement of Ethics}

The patient has given his written informed consent to publish his case including publication of images. The research was conducted ethically in accordance with the World Medical Association Declaration of Helsinki.

\section{Conflict of Interest Statement}

The authors have no conflicts of interest to declare.

\section{Funding Sources}

The study was financially supported by the Medical University of Lodz, Grant No. 503/1151-02/503-01.

\section{Author Contributions}

M.T. and K.M.-S. contributed equally to the preparation of this manuscript. K.M.-S. was responsible for the management of the patient. M.N. helped in the writing and editing of the original version of the manuscript and its revision.

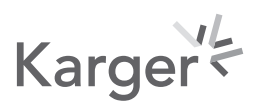




\section{References}

1 Searvance K, Jackson J, Schenkman N. Spontaneous perforation of the UPJ: a case report and review of the literature. Urol Case Rep. 2016 Nov 26;10:30-2.

2 Garg PK, Mohanty D, Rathi V, Jain BK. Spontaneous rupture of the renal pelvis presenting as an urinoma in locally advanced rectal cancer. World J Clin Cases. 2014 Apr 16;2(4):108-10.

3 Porfyris 0, Apostolidi E, Mpampali A, Kalomoiris P. Spontaneous rupture of renal pelvis as a rare complication of ureteral lithiasis. Turk J Urol. 2016 Mar;42(1):37-40.

4 Gershman B, Kulkarni N, Sahani DV, Eisner BH. Causes of renal forniceal rupture. BJU Int. 2011;108:1909-12.

5 Te-Li C, Yu-Jang S, Lap-Ming T, Wen-Han C, Chang-Chin C. Spontaneous rupture of renal pelvis. Int J Gerontol. 2007 Sep;1(3):131-3.

6 Main JM. Spontaneous rupture of the renal pelvis in the only functioning kidney. Br J Surg. 1967;54:1033-5 1967.

7 Allison MC, McLean L, Robinson LQ, Torrance CJ. Spontaneous urinoma due to retroperitoneal fibrosis and aortic aneurysm. Br Med J. 1985 Jul 20;291(6489):176.

8 Höltl G, Figdor PP. Spontaneous rupture of the renal collecting system. Int Urol Nephrol. 1975;7:109.

9 Ashebu S, Elshebiny Y, Dahniya M. Spontaneous rupture of the renal pelvis. Australas Radiol. 2000;44:125-7.

10 Wystrychowski A, Nowicki M, Kokot F. Hyponatraemic renal pseudofailure-don't forget the possibility of uroperitoneum. Nephrol Dial Transplant. 1996;11:2491-2.

11 Rosenzweig B, Pinthus JH, Kleinmann N, Joffe E, Erlich T, Fridman E, et al. The relative contribution of urine extravasation to elevate plasma creatinine levels in acute unilateral ureteral obstruction. Can Urol Assoc J. 2015 Jul-Aug;9(7-8):E428-33.

12 Yanaral F, Ozkan A, Cilesiz NC, Nuhoglu B. Spontaneous rupture of the renal pelvis due to obstruction of pelviureteric junction by renal stone: a case report and review of the literature. Urol Ann. 2017 Jul-Sep; 9(3):293-5. 